\title{
Molecular Biological Method Can Indicate Evolution of Xenobiotic Degraders from Activated Sludge
}

\author{
Nyuk-Min Chong \\ Department of Environmental Engineering, DaYeh University \\ No. 168, University Road, Dacun, Changhua, Taiwan 51591, R.O.C. \\ chong@mail.dyu.edu.tw
}

\section{Extended Abstract}

Activated sludge treatment of a xenobiotic organic compound requires acclimation to gain degradation capability. Activated sludge biomass after acclimation is thereby containing degraders capable of xenobiotic breakdown and nondegrader living on downstream metabolites. How degraders evolve from the process of acclimation is important to understanding xenobiotic degradation mechanisms. When these mechanisms are well understood and properly managed, technology of xenobiotic pollutant treatment can be much improved.

Various methods were used to identify xenobiotic degraders within microbial reaction systems that were used to degrade an array of xenobiotic organic compounds [1 5]. In this study, the molecular biology method of bacterial 16S rRNA pyrosequencing was employed to examine the change of activated sludge community structures before and after the sludge had acclimated to a target xenobiotic. The sample xenobiotic compound used as the activated sludge acclimation/degradation target was the herbicide 2,4-dichlorphenoxyacetic acid (2,4-D). Indigenous microorganisms in activated sludge seed was obtained from a soil that did not have any record of 2,4-D contamination. Acclimation reactions were performed in two (2) types of reactors with a feed medium containing essential nutrients and 2,4-D as the sole carbon source: 1) Batch reaction in which indigenous activated sludge was to fully acclimated to 2,4-D; 2) Continuous flow reactors (CSTR) operated to steady state at which 2,4-D was completely degraded.

Pyrosequencing results allowed analyses of community changes after activated sludge had acclimated to target xenobiotic 2,4-D. In taxonomic level of family, the most obvious degrader-containing bacterial families were found to be Alcaligenaceae, Sphingomonadaceae, Burkholderiaceae and Comamonadacea, in an approximate descending order of importance. Bacterial genera that increased in abundance served as the method for identifying the genera that had evolved as degraders of the xenobiotic. The genera that increased in abundance were checked with literature or degradative pathway databases to confirm those strains that are commonly seen as potent degraders of 2,4-D.

The final compositions of acclimated activated sludge communities were seen to make up with degraders and nondegraders, with degraders that could evolve degradative genes prior or simultaneous to metabolizing the xenobiotic for growth, contributing biogenic metabolites for the maintenance of non-degraders. The final compositions of degrader communities were made up with those that match the growth rate requirement set by the reactor system: In batch acclimation reaction, survival pressure selects 2,4-D degrader genera with species that are able to evolve or acquire degradative genes most efficiently or quickly. In continuous flow acclimation reactors, growth rate set by the reactor system selects the dominating degrader species that have the coping growth rate.

Molecular biological means of bacterial 16S rRNA sequencing, with some input of biochemical and genetic analyses, can be used to identify degrader and an initial and final community change can identify degrader evolution in activated sludge after the process of acclimation to degrade the target xenobiotic.

\section{References}

[1] X. Q. Chi, J. J. Zhang, S. Zhao, and N. Y. Zhou, "Bioaugmentation with a consortium of bacterial nitrophenol-degraders for remediation of soil contaminated with three nitrophenol isomers," Environmental Pollution, vol. 172, pp. 33-41, 2013.

[2] K. C. Cho, D. G. Lee, H. Roh, M. E. Fuller, P. B. Hatzinger, and K. H. Chu, "Application of 13C-stable isotope probing to identify RDX-degrading microorganisms in groundwater," Environmental Pollution, vol. 178, pp. 350-360, 2013. 
[3] Y. R. Luo, Y. Tian, X. Huang, C. L. Yan, H. S. Hong, G. H. Lin, and T. L. Zheng, "Analysis of community structure of a microbial consortium capable of degrading benzo( $\square$ )pyrene by DGGE," Marine Pollution Bulletin, vol. 58, no. 8, pp. 1159-1163, 2009.

[4] N. M. Chong and H. W. Chang, "Plasmid as a measure of microbial degradation capacity for 2,4-dichlorophenoxyacetic acid," Bioresource Technology, vol. 100, no. 3, pp. 1174-1179, 2009.

[5] N. M. Chong and T. Y. Lin, "Measurement of the degradation capacity of activated sludge for a xenobiotic organic," Bioresource Technology, vol. 98, no. 5, pp. 1124-1127, 2007. 